\title{
Toxoplasma gondii ROP17 promotes autophagy via the Bcl- 2-Beclin 1 pathway
}

\author{
Min Guo ${ }^{1,2,4}$, Jia Sun ${ }^{1,4}$, Wen-tao Wang ${ }^{1,4}$, Hong-yan Liu ${ }^{1,3}$, Yue-hua Liu ${ }^{1}$, Ke-ru Qin ${ }^{1}$, Jin-rui Hu ${ }^{1}$, Xin-yang Li ${ }^{1}$, \\ Hong-li Liu ${ }^{1}$, Wei Wang ${ }^{1}$, Zhao-yang Chen $^{2 * \mathbb{D}}$, Chun-fang Wang ${ }^{2 *(\mathbb{D})}$ and Hai-long Wang ${ }^{1^{*}(\mathbb{D})}$
}

\author{
${ }^{1}$ School of Basic Medicine, Basic Medical Science Center, Shanxi Medical University, Jinzhong, Shanxi, China; \\ ${ }^{2}$ Labratory Animal Center, Shanxi Key Laboratory of Experimental Animal Science and Animal Model of Human Disease, Shanxi \\ Medical University, Taiyuan,Shanxi, China; \\ ${ }^{3}$ Eugenics and Molecular Medicine Testing Center, Ulanqab Central Hospital, Wulanchabu, Neimenggu, China \\ ${ }^{4}$ Min Guo, Jia Sun and Wen-tao Wang contributed equally to this work
}

\begin{abstract}
The apicomplexan Toxoplasma gondii (Nicolle et Manceaux, 1908) secretes a group of serine/threonine kinases from rhoptries, which play vital roles in boosting intracellular infection. Toxoplasma gondii rhoptry organelle protein 17 (ROP17) is one of these important kinase proteins. Nevertheless, its function remains unclear. Here, we showed that ROP17 induced autophagy in vitro and in vivo. The autophagy of small intestine tissues of $T$. gondii tachyzoite (RH strain)-infected mice was detected by the immunohistochemistry staining of LC3B, Beclin 1 and P62. ROP17 overexpression augmented starvation-induced autophagy in HEK 293T cells as measured by MDC staining, transmission electron microscopy (TEM), fluorescence microscopy and Western blot analysis. Moreover, the interaction of ROP17 and Bcl-2 was confirmed using co-immunoprecipitation analysis, and the data demonstrated that ROP17 had an autophagic role dependent on the Beclin 1-Bcl-2 pathway, which was also revealed in an in vivo model through immunohistochemical staining. Pearson coefficient analysis showed that there existed strong positive correlations between the expression of ROP17 and LC3B, Beclin 1 and phosphorylation of Bcl-2, while strong negative correlations between the expression of ROP17 and p62 and Bcl-2. Collectively, our findings indicate that ROP17 plays a pivotal role in maintaining $T$. gondii proliferation in host cells via the promotion of autophagy-dependent survival.
\end{abstract}

Keywords: Toxoplasma, rhoptry organelle protein 17, cellular autophagy, Becn 1, B-cell lymphoma-2

Toxoplasma gondii (Nicolle et Manceaux, 1908) is an obligate intracellular parasite capable of infecting various warm-blooded animals, including avian and mammalian species, with approximately one-third of the global human population being infected (Saeij and Frickel 2017). Most people infected with $T$. gondii are asymptomatic; however, severe diseases such as encephalitis are shown in immunocompromised patients (Montoya and Liesenfeld 2004). Toxoplasma gondii has high proliferation and mobile potential and can spread among different host tissues and body fluids (Kaye 2011). This parasite invades the central nervous system (CNS) by hijacking leukocytes to use them as shuttles, which enables extravasation across the bloodbrain barrier (BBB) (Santiago-Tirado and Doering 2017). In addition, dendritic cells (DC) and monocytes are important in T. gondii invading CNS (Sanecka and Frickel 2012, Schneider et al. 2019).

Tachyzoites of $T$. gondii proliferate in parasitophorous vacule and secrete a large set of proteins, including dense granule proteins (GRAs) and rhoptry organelle proteins (ROPs), which resist host cell functions. These proteins reach the host cytosol through either direct injection or translocation across the parasitophorous vacuolar membrane (PVM) (Panas et al. 2019). Most known ROPs are phosphokinases that belong to the serine/threonine (S/T) kinase family, ROP2 (El Hajj et al. 2006). ROP2-like kinase ROP18 has been extensively studied for its defence mechanism against immunity-related GTPases (IRGs) that are generated in response to up-regulation of IFN-gamma to kill the parasites in PVM (Taylor et al. 2006, El Hajj et al. 2007, Hermanns et al. 2016). ROP17, serine/threonine protein kinase that resides on the host cytosol side of the PVM in infected cells, has previously been shown to phosphorylate and thereby inactivate host immunity-related GTPase in collaboration with ROP18 and ROP5 (Etheridge et al. 2014). However, detail function of ROP17 remains unknown. 
Autophagy is a response to conserved cell-autonomous catabolic stress dedicated to the breakdown of cellular material and cell content recycling. Apart from its homeostatic role, autophagy is involved in the clearance of pathogens and the provision of nutrients for pathogen survival (Gomes and Dikic 2014). Infection with T. gondii leads to parasite death via autophagy when activated by CD40, rapamycin or when the pathways utilised by the parasite to subvert autophagic targeting are inhibited (Van Grol et al. 2013, Subauste 2019). However, the parasite can also take advantage of autophagy in host cells to promote their proliferation (Gao et al. 2014). In order to reveal the modulatory role of ROP17 on autophagy, autophagy in the small intestine of $T$. gondii-infected mice was analysed. In vitro assay, an ROP17-containing eukaryotic expression plasmid was transfected into HEK 293 T cells, and the autophagy of the transfected cells was assessed. Furthermore, we identified the intracellular signalling pathways involved in the ROP17-mediated activation of autophagy.

\section{MATERIALS AND METHODS}

\section{Preparation of rabbit anti-Toxoplasma gondii ROP17 serum}

Tachyzoites of the virulent $T$. gondii $\mathrm{RH}$ strain were maintained by intraperitoneal passage in BALB/c mice (from the Institute of Laboratory Animal Science of the Chinese Academy of Medical Science, Beijing, China) and were collected from peritoneal exudates $72 \mathrm{~h}$ after infection with $1 \times 10^{3}$ tachyzoites. Parasites obtained from the peritoneal exudate were washed three times with PBS solution by centrifugation at $1,000 \mathrm{~g}$ and resuspended in PBS.

Purified T. gondii ROP17 protein (TgROP17) was prepared according to our previous study (Wang et al. 2014) and the antiserum against $T g R O P 17$ was produced in two male New Zealand rabbits (body weight: $\sim 2.0-2.5 \mathrm{~kg}$ ). Before challenge, a 5 $\mathrm{ml}$ peripheral blood sample was collected from each rabbit ear vein for the preparation of a pre-immune or negative control. The TgROP17 protein $(500 \mu \mathrm{g})$ was intradermally injected into the backs of the rabbits, and the same dose was used in four boosters administered every 10 days thereafter. Blood was taken from the marginal ear vein 10, 20, 30 and 40 days after the first challenge and on the 10th day after the final challenge. In addition, a blood sample was obtained from the jugular vein for detecting of $T$. gondii-specific antibody using enzyme-linked immunosorbent assay (ELISA) with $\operatorname{TgROP} 17$ used as a coating antigen. The serum phase was obtained by centrifugation separation at 2,300 $\mathrm{g}$ for 10 minutes at $4{ }^{\circ} \mathrm{C}$. After removing the precipitated residues, $0.02 \%$ $\mathrm{NaN}_{3}$ was added to the blood serum, which was stored at $-80^{\circ} \mathrm{C}$. All experimental animal procedures were approved by the Ethics Committee of Animal Experiments of Shanxi Medical University (permit Nos. 20110320-1 for mice experiment and 20180206-1 for rabbit experiment).

The titre of the ROP17 antiserum was detected by direct coating ELISA according to the published method (Chen et al. 2004). Ninety six microtitre plate (Corning, Corning, NY, USA) was coated with $100 \mathrm{ng} / 100 \mu 1$ of purified GST-ROP17, and blocked with PBST containing 1\% bovine serum albumin (BSA) for $2 \mathrm{~h}$ at $37{ }^{\circ} \mathrm{C}$. The anti-ROP17 polyclonal antiserum was diluted to different concentrations $(1: 1,000 ; 1: 2,000 ; 1: 4,000 ; 1: 8,000$;
$1: 16,000 ; 1: 32,000 ; 1: 64,000 ; 1: 128,000 ; 1: 256,000 ;$ and 1 : 512,000$)$ with $1 \%$ BSA and incubated for $2 \mathrm{~h}$ at $37^{\circ} \mathrm{C}$. Then, the plate was incubated for $1 \mathrm{~h}$ with goat anti-rabbit IgG-HRP (Sigma, Burlington, MA, USA) diluted to $1: 2,000$ with $1 \%$ BSA. Tetramethylbenzidine (TMB) chromogenic reagent (Boster, Wuhan, China) was added to the plate that had been washed with TBST. After $50 \mathrm{ml}$ of $2 \mathrm{M} \mathrm{H}_{2} \mathrm{SO}_{4}$ was added to plates to stop the reaction. The absorbance was measured at $450 \mathrm{~nm}$ by an ELISA plate reader (Epoch Multi-Volume Spectrophotometer System, BioTek, Winooski, USA).

\section{Immunohistochemistry}

The small intestine tissues of $T$. gondii tachyzoites-infected mice or normal mice were collected in our previous study (Wang et al. 2016) and used for immunohistochemistry assay. The small intestine was stored overnight in $4 \%$ paraformaldehyde at $4{ }^{\circ} \mathrm{C}$. The $5 \mu \mathrm{m}$ thick sections were prepared and incubated overnight at $4{ }^{\circ} \mathrm{C}$ with ROP17 (dilution $1: 200$ ), LC3B, Beclin 1, p62, Bcl2 and phospho-Bcl-2 (Ser70) antibodies (dilution $1: 100)$ after the endogenous peroxidase activity was blocked, followed by incubation with horseradish peroxidase-coupled goat anti-rabbit secondary antibody at $37^{\circ} \mathrm{C}$ for $1 \mathrm{~h}$ and staining with 3,3'-diaminobenzidine. The samples incubated with rabbit serum instead of indicated primary antibodies were used as negative control. One hundred cells were captured randomly in a field of view under the microscope (CX33, OLYMPUS, Tokyo, Japan) and the percentage of the positive cells was calculated.

\section{Cell culture and transfection}

HEK 293 T cells were purchased from the Cell Culture Center of the Chinese Academy of Medical Sciences (Beijing, China) and cultured in high-glucose Dulbecco's modified Eagle's medium (DMEM; Gibco, Grand Island, NY, USA). The medium was supplemented with $10 \%$ (v/v) foetal bovine serum (FBS; Gibco, USA). The cells were cultured at $37{ }^{\circ} \mathrm{C}$ in an $5 \% \mathrm{CO}_{2}$ incubator. The cells were passaged every $\sim 2-3$ days when they reached $\sim$ $70-80 \%$ confluency.

HEK 293 T cells cultured in a 6 -well plate $\left(2.5 \times 10^{5}\right.$ cells/well, $1 \mathrm{ml} \mathrm{medium} /$ well) were transfected with $\mathrm{p} 3 \times$ Flag-CMV-14 and p3 $\times$ Flag-CMV-14-TgROP17 using Lipofectamine 3000 (Thermo Fisher Scientific, Waltham, MA, USA) according to the manufacturer's instructions. Forty-eight hours later, the cells were treated with serum-free medium and cultured for another $4 \mathrm{~h}$ and $8 \mathrm{~h}$ to induce autophagy.

\section{Monodansylcadaverine (MDC) staining by fluorescent micro- scope}

The transfected HEK 293T cells were treated by serum starvation for 0,4 and $8 \mathrm{~h}$, and then, the cells were incubated with MDC $(50 \mu \mathrm{mol} / \mathrm{l})$ at $37^{\circ} \mathrm{C}$ for 30 minutes. Then, the cells were washed two times with pre-cooled PBS and immediately observed and imaged under a fluorescence microscope (EVOS M5000, Thermo Fisher Scientific, USA). One hundred cells per sample were analysed, and the percentage of autophagic cells was calculated.

\section{Transmission electron microscopy (TEM)}

After serum starvation treatment, the HEK 293T cells were collected and washed with cold PBS, fixed overnight in 3\% glutaraldehyde at $4{ }^{\circ} \mathrm{C}$ and subsequently post-fixed in $1 \%$ osmium 
tetroxide for 30 minutes. The cells were gradient dehydrated with ethanol solutions ranging from $50 \%$ to $100 \%$ in a $10 \%$ graded series and then embedded in Eponate 12 resin (Ted Pella, Redding, CA, USA). The blocks were cut into ultrathin sections and then twice stained with uranyl acetate and lead citrate. The cells were observed under a transmission electron microscope (JEOL-100CX, Tokyo, Japan).

\section{Examination of GFP-LC3B puncta}

HEK $293 \mathrm{~T}$ cells were co-transfected with $\mathrm{p} 3 \times$ Flag-CMV-14 or p3 $\times$ Flag-CMV-14-TgROP17 and pCMV-GFP-LC3 (GenePharma Co., Ltd, China). After 48 h, the cells were cultured in serum-free medium for $4 \mathrm{~h}$ and $8 \mathrm{~h}$. GFP-LC3 puncta were quantified by observation with a fluorescence microscope (EVOS M5000, Thermo Fisher Scientific) and counting at least 300 cells. The results are plotted as the mean $\pm \mathrm{SD}$ of three independent experiments.

\section{Co-immunoprecipitation}

Co-immunoprecipitation analysis was performed in accordance with the manufacturer's instructions. Briefly, HEK 293T cells were transfected with $\mathrm{p} 3 \times$ Flag-CMV-14 or $\mathrm{p} 3 \times$ Flag-CMV14- $T g R O P 17$ for $48 \mathrm{~h}$, and then, the cells were harvested and lysed using sonication in cell lysis buffer (50 mM Tris-HCl, $\mathrm{pH} 7.5$; $150 \mathrm{mM} \mathrm{NaCl} ; 1 \%$ Nonidet P-40; $0.5 \%$ sodium deoxycholate; and a $1 \%$ protease inhibitor cocktail; Roche, Basel, Switzerland). The cell lysates were centrifuged and the supernatant was incubated overnight with FLAG M2 Affinity Gel (No. A2220, Sigma-Aldrich, USA) with gentle agitation at $4{ }^{\circ} \mathrm{C}$. The following day, the beads were washed six times with cell lysis buffer and heated in $2 \times$ sample buffer. After brief centrifugation, the supernatant was used for Western blotting.

\section{Western blotting}

The protein from $1 \times 10^{6}$ tachyzoites of $T$. gondii (Ma et al. 2009) was prepared using $1 \times$ SDS loading buffer heated for 10 minutes. Total protein from the HEK $293 \mathrm{~T}$ cells was extracted using RIPA buffer with protease inhibitors and quantified using BCA protein assay reagent (Thermo Scientific, USA). Equal amounts of protein samples were used for Western blotting assay. The ECL blot detection system (Transgene, Beijing, China) with a Bio-Rad Universal Hood II Gel Doc XR system was used for calculating the target protein level. $\beta$-actin on the same membrane was used as an internal control. The following antibodies were used in this study: rabbit monoclonal anti-LC3B (No. 3868, Cell Signaling Technology, Boston, USA), rabbit monoclonal anti-Beclin-1 (No. 3495, Cell Signaling Technology), rabbit monoclonal anti-p62 (No. 39749, Cell Signaling), rabbit monoclonal anti-Flag tag (No. 14793, Cell Signaling), rabbit monoclonal anti-phospho-Bcl-2 (Ser70) (No. 2827 Cell Signaling), rabbit polyclonal anti-JNK (No. 9252, Cell Signaling), rabbit monoclonal anti-phospho-SAPK/JNK (Thr183/Tyr185) (No. 4668), Cell $\mathrm{r}$ and rabbit monoclonal $\beta$-actin (No. 4970, Cell Signaling). Horseradish peroxidase (HRP)-linked anti-rabbit IgG secondary antibodies (No. 7074, Cell Signaling) were also used.

\section{Statistical analysis}

Data are presented as means \pm standard deviation (SD). Differences between two groups were analysed using Student's $t$-test.
Pearson coefficient was used to analyse correlations between the expression of ROP17 with the expression of autophagy markers LC3B, beclin 1 and $\mathrm{p} 62$, and with phosphorylation of Bcl-2 and $\mathrm{Bcl}-2$. An $r$-value $\geq 0.5$ was considered as strong correlation, and an $r$-value $<0.5$ was considered as poor correlation (Hassan et al. 2018). A $p$-value $<0.05$ was considered as statistically significant. All data were analysed using SPSS software version 19.0 (SPSS Inc., Chicago, IL, USA).

\section{RESULTS}

\section{Rabbit anti-ROP17 antiserum was efficient for detecting ROP17 expression}

The titreof rabbit anti-ROP17 polyclonal antiserum was 1 : 128,000 (Fig. 1a and Table 1). To confirm the immunoreactivity of the ROP17 polyclonal antiserum, Western blotting was employed to detect the purified GST-ROP17 and tachyzoite protein of Toxoplasma gondii using rabbit anti-ROP17 polyclonal antiserum as the primary antibody. As illustrated in Fig. 1b, specific bands of approximately 96 $\mathrm{kDa}$ (GST-ROP17) and $69 \mathrm{kDa}$ (ROP17, from tachyzoite of $T$. gondii) were detectable, indicating that the rabbit anti-ROP17 antibody was prepared successfully.

\section{Tachyzoites of $T$. gondii induce autophagy in small intestinal cells}

To determine whether autophagy occurs in tachyzoite-infected murine cell models, paraffin-embedded sections of the small intestine were prepared for the immunohistochemical detection of autophagy markers. As shown in Fig. 2, the levels of LC3B $(21 \pm 4 \%$ and $37 \pm 5 \%$ positive cell rate in $24 \mathrm{~h}$ and $48 \mathrm{~h}$ collected tissues, respectively) and beclin 1 (32 $\pm 6 \%$ and $45 \pm 8 \%$ positive cell rate in

a

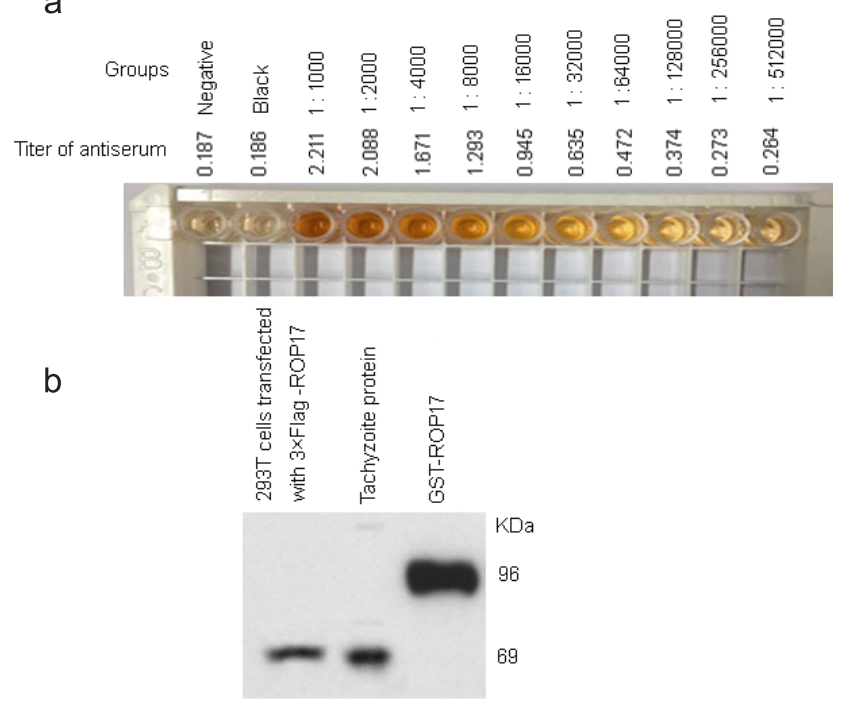

Fig. 1. The rabbit anti-ROP17 antiserum was evaluated using direct-coated ELISA (a) and Western blotting (b). The rabbit serum added before immunisation served as the negative control, and $1 \%$ BSA was used as the blank control, as shown in Fig. 1a. The lysate from the HEK 293T cells which were transfected with $\mathrm{p} 3 \times$ Flag-CMV-14-ROP17 for $48 \mathrm{~h}$ and $1 \mu \mathrm{g}$ of purified GST-ROP17 were used as positive controls to assess the expression of ROP17 in the tachyzoites of Toxoplasma gondii (Nicolle et Manceaux, 1908) as shown in Fig. 1b. 
Table 1. The titre of rabbit anti-ROP17 polyclonal antiserum $(n=3)$

\begin{tabular}{|c|c|c|c|c|c|c|c|c|c|c|c|c|}
\hline Groups & Negative & Blank & $1: 1,000$ & $1: 2,000$ & $1: 4,000$ & $1: 8,000$ & $1: 16,000$ & $1: 32,000$ & $1: 64,000$ & $1: 128,000$ & $1: 256,000$ & $1: 512,000$ \\
\hline $\mathrm{OD}_{450}$ & $\begin{array}{c}0.188 \pm \\
0.007\end{array}$ & $\begin{array}{c}0.186 \pm \\
0.009\end{array}$ & $\begin{array}{c}2.211 \pm \\
0.021^{*}\end{array}$ & $\begin{array}{c}2.088 \pm \\
0.019^{*}\end{array}$ & $\begin{array}{c}1.671 \pm \\
0.018^{*}\end{array}$ & $\begin{array}{c}1.293 \pm \\
0.011^{*}\end{array}$ & $\begin{array}{c}0.946 \pm \\
0.013^{*}\end{array}$ & $\begin{array}{c}0.635 \pm \\
0.071^{*}\end{array}$ & $\begin{array}{c}0.472 \pm \\
0.041^{*}\end{array}$ & $\begin{array}{c}0.375 \pm \\
0.032^{*}\end{array}$ & $\begin{array}{c}0.273 \pm \\
0.016\end{array}$ & $\begin{array}{c}0.264 \pm \\
0.013\end{array}$ \\
\hline
\end{tabular}

Negative group: Pre immune serum. " means $\mathrm{p}<0.05$ compared with negative group.

a

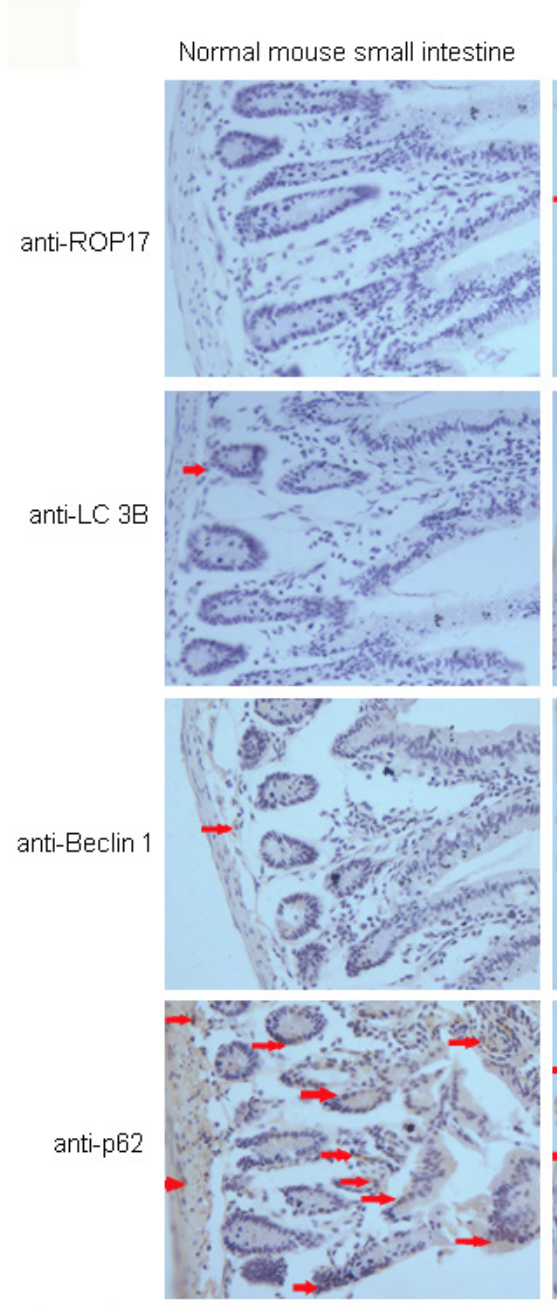

b

\section{$24 \mathrm{~h}$ after tachyzoites}

of $T$, gondii infection
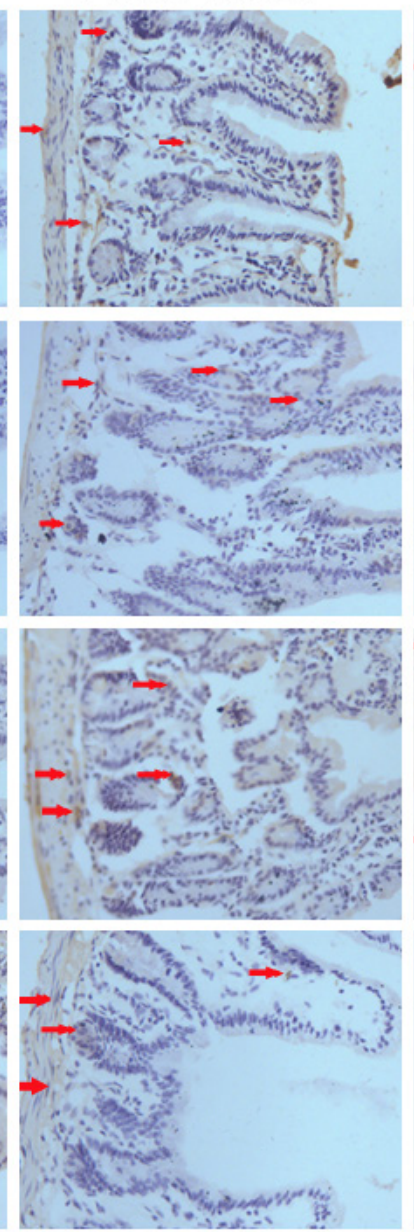

$48 \mathrm{~h}$ after tachyzoites

of $T$, gondii infection
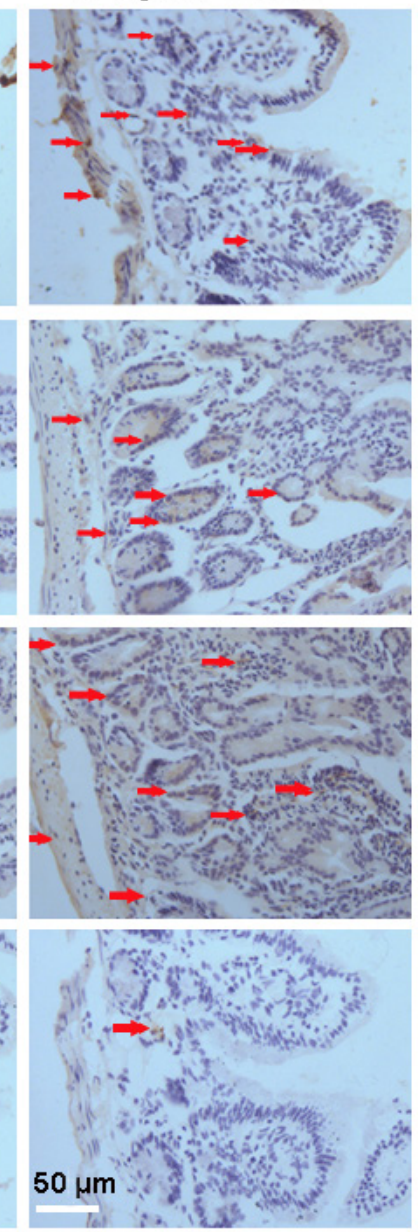

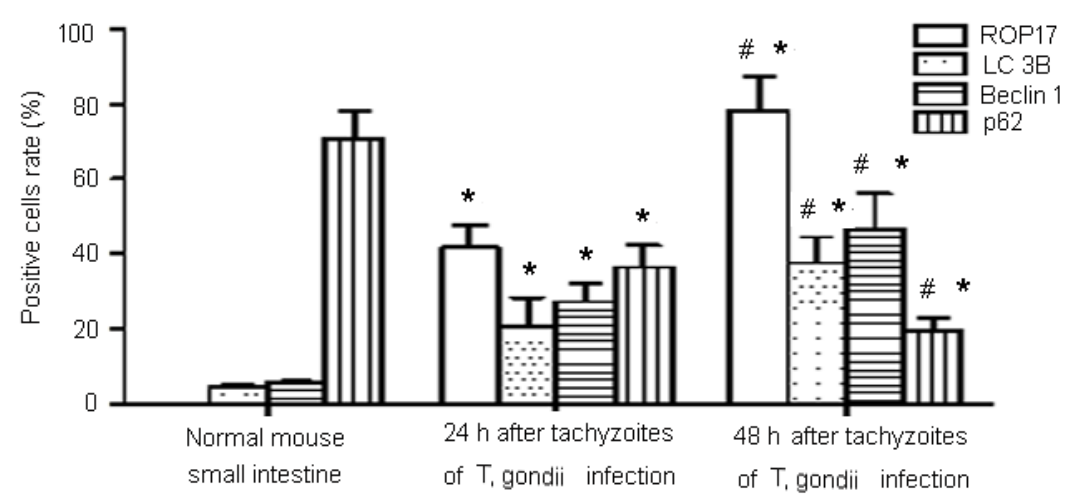

Fig. 2. Tachyzoites of Toxoplasma gondii (Nicolle et Manceaux, 1908) induced autophagy in the mouse small intestine cells (a and b). 

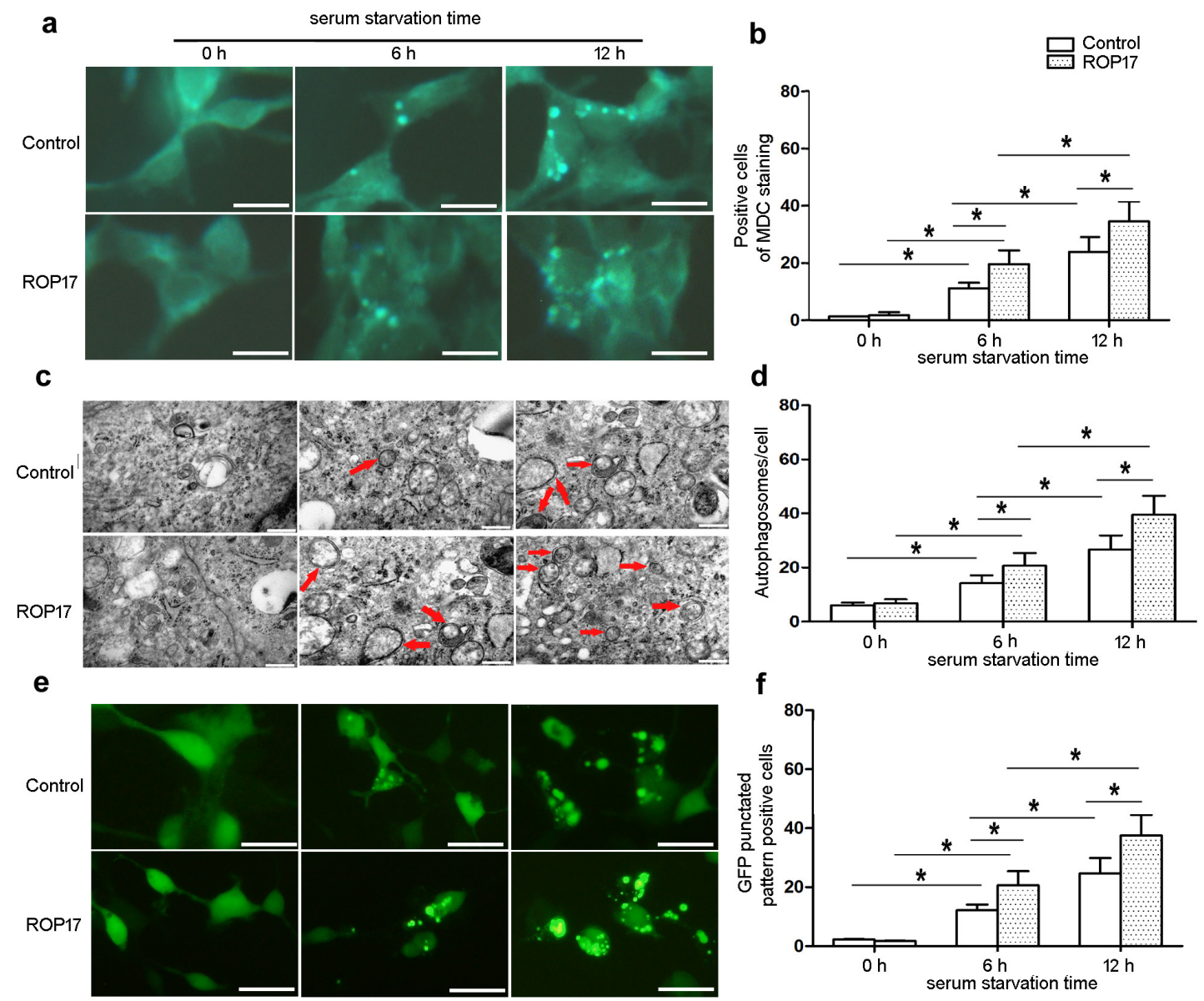

Fig. 3. ROP17 enhanced the autophagy of HEK $293 \mathrm{~T}$ cells subjected to serum starvation. a and b are MDC staining, autophagic vacuoles of the ROP17-overexpressing 293T cells and control cells were visualised by MDC staining after serum starvation for 0, 6 and 12 $\mathrm{h}$, the scale bar represents $20 \mu \mathrm{m}$. $\mathrm{c}$ and $\mathrm{d}$ are transmission electron microscopy analysis of the serum-starved ROP17-overexpressing $293 \mathrm{~T}$ cells and control cells. ROP17-overexpressing cells displayed more autophagosomes (indicated by red arrows) than the controls. The relative numbers of autophagosomes in the different groups are shown (d). The scale bar represents $500 \mathrm{~nm}$. e and $\mathrm{f}$ are accumulation of GFP-LC3 puncta. The 293 T cells were co-transfected with p $3 \times$ Flag-CMV-14 or p3 $\times$ Flag-CMV-14-TgROP17 and GFP-LC3 and subjected to serum starvation for 0,6 and $12 \mathrm{~h}$. GFP-LC3B puncta were observed by confocal laser scanning microscopy (e), and showed a punctuated pattern (f), indicating the induction of autophagy. The scale bar represents $20 \mu \mathrm{m}$. The data are expressed as the mean \pm S.D. of three independent experiments. *Indicates a significant difference between groups as shown, ${ }^{*} p<0.05$.

$24 \mathrm{~h}$ and $48 \mathrm{~h}$ collected tissues) increased compared with those in normal tissues ( $4 \pm 1 \%$ and $5 \pm 1 \%$ ), while that of p62 decreased gradually with prolonged time of infection by the tachyzoites of $T$. gondii $(24 \pm 4 \%$ and $17 \pm 3 \%$ positive cell rate in $24 \mathrm{~h}$ and $48 \mathrm{~h}$ collected tissues) compared with that in nomal tissues $(72 \pm 4 \%)$. Notably, the number of tachyzoites of $T$. gondii was also augmented upon enhanced autophagy, as illustrated by ROP17-positive staining. These results clearly showed that the infection by the tachyzoites of $T$. gondii via the intraperitoneal injection triggered autophagy in mouse small intestinal cells. However, whether the proliferation of tachyzoites depends on autophagy still remains unclear and need to be further investigated.

\section{The overexpression of ROP17 promotes the autophagy} induced by serum starvation

To evaluate the putative roles of ROP17 in T. gondii tachyzoite and host cell interplay, we used the HEK $293 \mathrm{~T}$ cell line. Transient transfection of the p3 $\times$ Flag-CMV-14 or p3 $\times$ Flag-CMV-14-TgROP17 eukaryotic expression plasmid for $48 \mathrm{~h}$ and the expression of ROP17-Flag was confirmed by Western blotting (Fig. 1b). We examined the initiation of autophagy in HEK $293 \mathrm{~T}$ cells with or without ROP17 after serum starvation for $0 \mathrm{~h}, 6 \mathrm{~h}$ and $12 \mathrm{~h}$ by MDC staining. To distinguish the role of ROP17 in autophagy, the HEK 293T cells were incubated with MDC (1 $\mathrm{mM}$ ) after serum starvation. The staining results indicated that the number of acidic vacuoles was increased in the ROP17-overexpressing HEK 293T cells $(21 \pm 2 \%$ at $6 \mathrm{~h}$ and $36 \pm 7 \%$ at $12 \mathrm{~h}$, respectively) compared with control cells ( $13 \pm 3 \%$ at $6 \mathrm{~h}$ and $24 \pm 4 \%$ at $12 \mathrm{~h}$ ) (Fig. 3a,b), suggesting that ROP17 possibly induced autophagy.

To confirm that ROP17 induced autophagy upon serum starvation, transmission electron microscopy (TEM) was used. As illustrated in Fig. 3c,d, the number of autophagosomes in the ROP17-overexpressing cells was increased $(20 \pm 3 \%$ at $6 \mathrm{~h}$ and $41 \pm 6 \%$ at $12 \mathrm{~h})$ than that of control $(16 \pm 2 \%$ at $6 \mathrm{~h}$ and $23 \pm 5 \%$ at $12 \mathrm{~h})$ without serum. ROP17-overexpressing HEK 293T cells and parental cells 


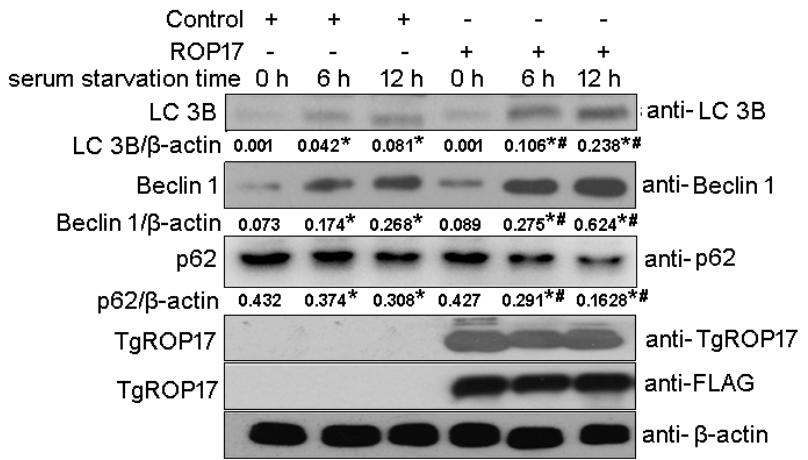

Fig. 4. ROP17 of Toxoplasma gondii (Nicolle et Manceaux, 1908) induced autophagy in serum-free ROP17-overexpressing HEK 293 cells. ROP17-induced autophagy, detected by the presence of Beclin 1, LC3B and p62, was analysed by Western blotting. $\beta$-actin served as a loading control. One representative of three independent experiments is shown. The quantitative results are presented as the ratio of $\mathrm{LC} 3 \mathrm{~B}$ to $\beta$-actin, Beclin 1 to $\beta$-actin and p62 to $\beta$-actin $(\mathrm{n}=3) .{ }^{*} p<0.05$ indicates a significant difference compared with the difference calculated for the previous time point, and $\# p<0.05$ indicates a significant difference compared with the value calculated for same time point in the control group.

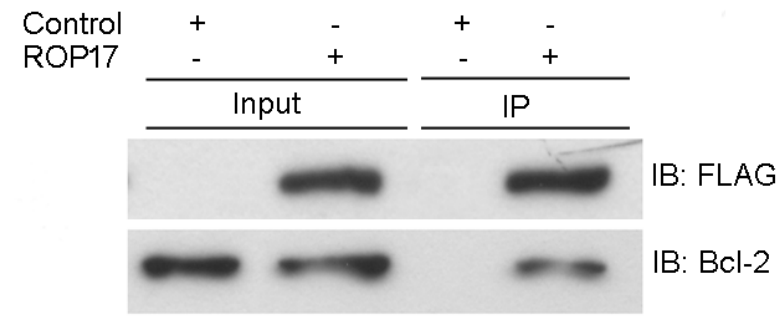

Fig. 6. A co-IP assay was employed to investigate the interaction of ROP17 of Toxoplasma gondii (Nicolle et Manceaux, 1908) and Bcl-2. HEK 293 T cells were transfected with p3 $\times$ Flag-CMV-14 or p3 $\times$ Flag-CMV-14-TgROP17, and the cell lysates were incubated with FLAG M2 Affinity Gel. The precipitate was assayed using Western blotting with Flag and Bcl-2 antibodies.

transfected with GFP-LC3 were subjected to serum starvation. A marked accumulation of GFP-LC3 puncta was observed in the ROP17-overexpressing HEK $293 \mathrm{~T}$ cells (23 $\pm 3 \%$ at $6 \mathrm{~h}$ and $35 \pm 6 \%$ at $12 \mathrm{~h}$ ) compared with control cells ( $11 \pm 2 \%$ at $6 \mathrm{~h}$ and $22 \pm 5 \%$ at $12 \mathrm{~h}$ ), suggesting that ROP17 led to autophagosome accumulation (Fig. 3e,f).

To confirm the role of ROP17 in autophagy induction, the autophagy markers LC-3B, Beclin 1 and p62 were analysed by Western blotting. As illustrated in Fig. 4, the levels of Beclin 1 and LC3B in the serum-starved ROP17-overexpressing HEK293T cells were greater than those in the control cells, while p62 showed the opposite trend at the same indicated times. All of these data suggest that ROP17 promotes the autophagy induced by serum starvation.

\section{TgROP17 phosphorylates Bcl-2 and promotes autophagy}

On western blotting, ROP17 enhanced the phosphorylation of Bcl-2 (Ser 70) and led to its degradation (Fig. 5). To determine whether phosphorylation is ROP17- or JNK-de-

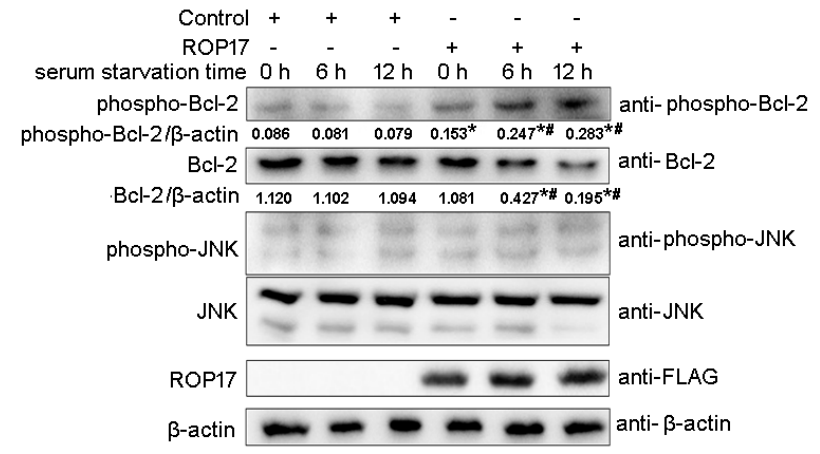

Fig. 5. ROP17 of Toxoplasma gondii (Nicolle et Manceaux, 1908) augmented autophagy via the beclin 1-Bcl-2 pathway. HEK 293T cells were transfected with $\mathrm{p} 3 \times$ Flag-CMV-14 or $\mathrm{p} 3 \times$ Flag-CMV14-TgROP17 and then subjected to serum starvation for $0 \mathrm{~h}, 6 \mathrm{~h}$ and $12 \mathrm{~h}$. The phosphorylation of Bcl-2 and Bcl-2, and the phosphorylation of JNK and JNK were tested by Western blotting. $\beta$-actin served as a loading control. One representative of three independent experiments is shown. The quantitative results are presented as the ratio of phosphorylation of $\mathrm{Bcl}-2$ to $\beta$-actin and $\operatorname{Bcl}-2$ to $\beta$-actin $(\mathrm{n}=3) ;{ }^{*} p<0.05$ indicates a significant difference compared with the value calculated for the previous time point, and $\# p<0.05$ indicates a significant difference compared with the value calculated for the same time point in the control group.

pendent, the phosphorylation of JNK was also measured as shown in Fig. 5; ROP17 did not change the levels of JNK or its phosphorylation. To exploit the function of ROP17 in $\mathrm{Bcl}-2$ phosphorylation, co-immunoprecipitation experiments were performed, and the data showed that ROP17 with Bcl-2 (Fig. 6). Collectively, these data demonstrate that ROP17 interacts with Bcl-2 and phosphorylates it and then activates autophagy.

\section{Infection with tachyzoites of $T$. gondii facilitates the phosphorylation and degradation of $\mathrm{Bcl}-2$ in the mouse small intestine cells}

Based on the results from the in vitro assay, we wanted to determine whether ROP17 functions as an autophagy inducer via the Beclin 1-Bcl-2 pathway in vivo. Mouse small intestine slices that had been used to analyse autophagy (Fig. 2) were collected to evaluate the levels of Bcl-2 and its phosphorylation. As illustrated in Fig. 7, the phosphorylation of Bcl-2 increased, but the Bcl-2 level was attenuated gradually according the time of infection with the tachyzoites of $T$. gondii.

To further confirm ROP17 as a cellular autophagy modulator, correlations between the expression of ROP17 with the expression of autophagy markers LC3B, beclin 1 and p62, as well as phosphorylation of Bcl-2 and $\mathrm{Bcl}-2$ were evaluated using Pearson's correlation coefficient. As shown in Fig. 8 , a strong positive correlation was illustrated between the expression of ROP17 with the expression of LC3B (Fig. 8a, $r=0.9826, p=0.0443$ ), Beclin 1 (Fig. 8b, $r=0.9945, p=$ 0.0334), and phosphorylation of Bcl-2 (Fig. $8 \mathrm{~d}, r=0.9908, p$ $=0.0229$ ), while a strong negative correlation was revealed between the expression of ROP17 and the expression of $\mathrm{p} 62$ (Fig. 8c, $r=-0.9735, p=0.0364$ ), and Bcl-2 (Fig. 8e, $r=$ $-0.9762, p=0.0301)$. These data together indicate that the 


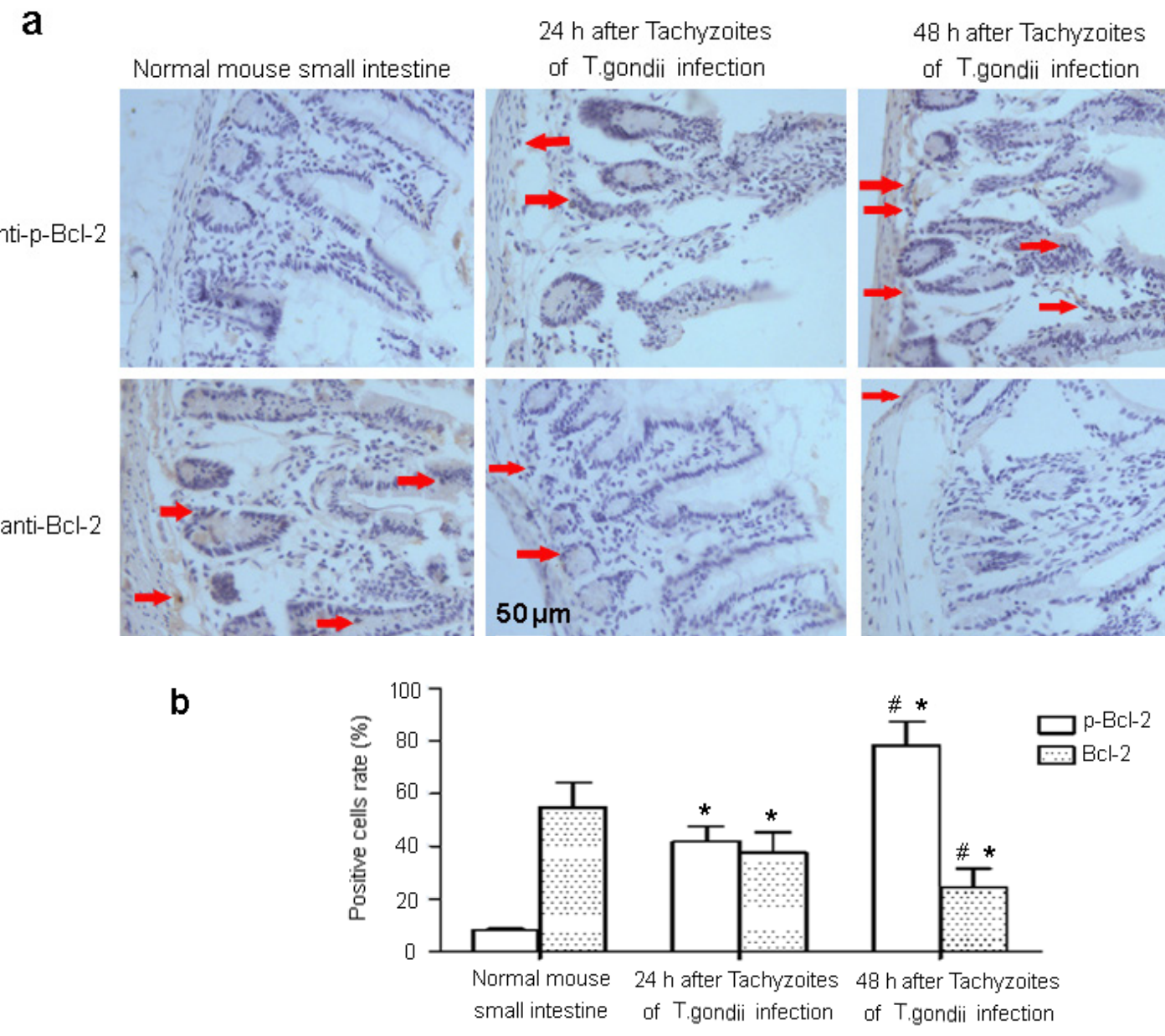

Fig. 7. Tachyzoites of Toxoplasma gondii (Nicolle et Manceaux, 1908) boosted the phosphorylation and degradation of Bcl-2 in the mouse small intestine. A total of 103 tachyzoites of T. gondii were injected into the mouse abdominal cavity, and the small intestine was collected $24 \mathrm{~h}$ and $48 \mathrm{~h}$ later for immunohistochemical analysis using Bcl-2 and phospho-Bcl-2 (Ser70) antibodies (a). The normal mouse small intestine served as the control. The positive expression rate of the target proteins was calculated (b). Data are expressed as the means $\pm \mathrm{SD}(\mathrm{n}=3)$. Red arrows indicate positive cells. The scale bar represented $50 \mu \mathrm{m}$ for all slices; $* p<0.05$ indicates a significant difference compared with the normal mouse group, and $\# p<0.05$ indicates a significant difference compared with the $T$. gondii infection for the $24 \mathrm{~h}$ group

increase of LC3B, Beclin 1 and phosphorylation of Bcl-2, the decrease of p62 and Bcl-2 are ROP17-modulated.

\section{DISCUSSION}

Autophagy is an evolutionarily conserved cell survival process that removes damaged organelles, toxic metabolites and pathogens and enhances energy production to maintain cell survival under nutrient-deficient conditions. In contrast, excessive self-digestion and degradation of important intracellular components can lead to cell death. Whether autophagy plays a pro-survival or pro-death role depends on the type and extent of the stress, cell context, etc. (Wirawan et al. 2012). For intracellular pathogens, host cells provide a replicative niche but also combat intruders via different responses. Among these responses, host autophagy is a significant hindrance to the intracellular growth of pathogens but can also be subverted by pathogens to capture nutrients that support their survival. However, the role of autophagy during infection is complex; some pathogens rely on the induction of host autophagy to survive within host cells, while others are destroyed by it. The dif- ferent roles of autophagy in eukaryotic pathogens rely on the host cell types they infect, infective stage and response strategies (Evans et al. 2018).

Under natural conditions, following host oral ingestion of tissue cysts or oocysts, the parasites invade the intestinal tissue and rapidly develop into tachyzoites (Dubey 1997a, b). Tachyzoites of Toxoplasma gondii infect virtually all nucleated cells and survive by residing in a compartment called the parasitophorous vacuole (PV), which is formed during the active invasion of host cells and is dependent on the parasite actin-myosin motor and sequential secretion of proteins from micronemes and rhoptries (Santos and Soldati-Favre 2011). Rhoptries are key secretory organelles that synthesise rhoptry organelle proteins (ROPs) and play essential roles in parasite survival (Hakimi et al. 2017). ROP16, ROP17 and ROP18 are serine/threonine $(\mathrm{S} / \mathrm{T})$ protein kinases secreted by rhoptries (Peixoto et al. 2010). ROP16 phosphorylates the host transcriptional factors STAT3 and STAT6 to negatively regulate the production of IL-12 and Th1 inflammatory responses, which are essential for the host to survive infection with $T$. gondii 


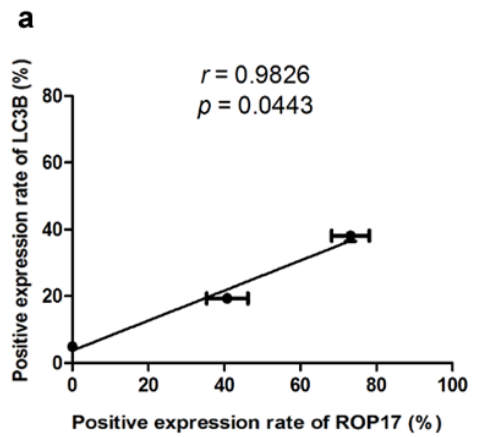

d

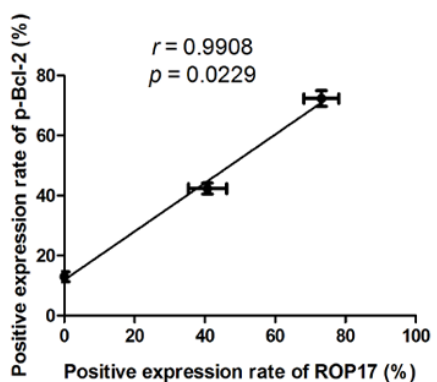

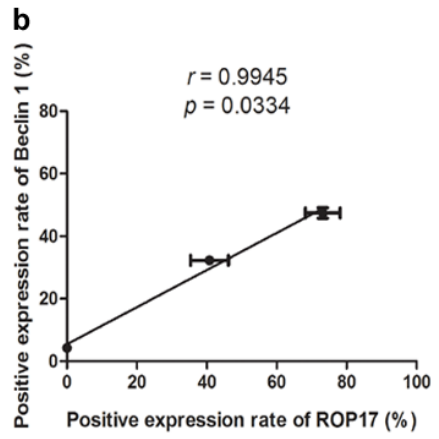
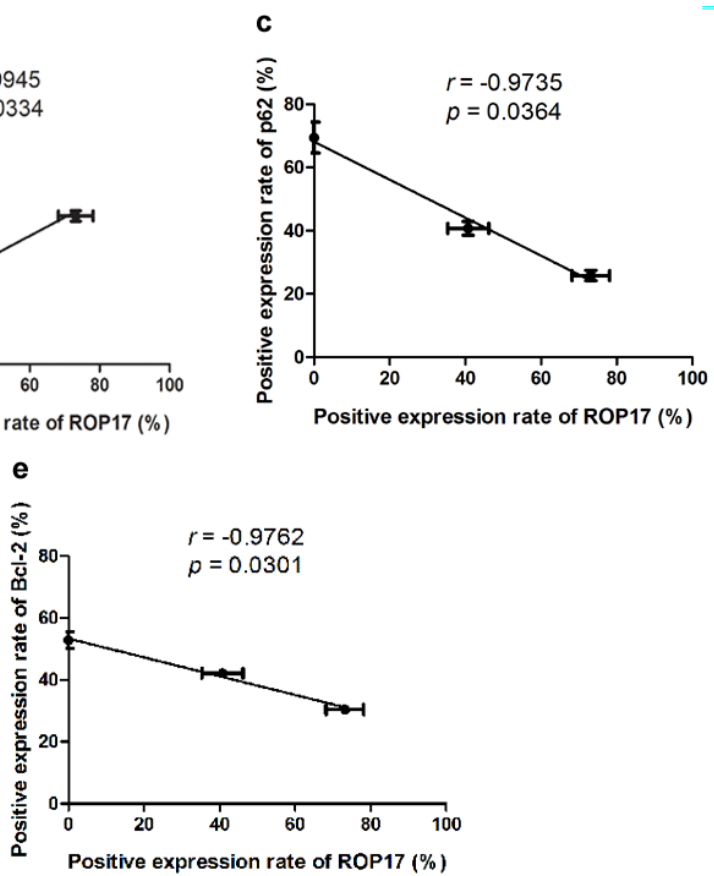

Fig. 8. Expression of ROP17 of Toxoplasma gondii (Nicolle et Manceaux, 1908) correlates with autophagy markers and Bcl-2. Immunohistochemistry assay were performed in small intestine tissue obtained from tachyzoites of $T$. gondii-infected mouse at $24 \mathrm{~h}$ and $48 \mathrm{~h}$ after the infection. ROP17, LC3B, Bcelin 1, p62, Bcl-2 and phosto-Bcl-2 (Ser70) were stained with corresponding antibodies. Normal mouse small intestine served as the control. The positive expression rate of target proteins was calculated, and the correlations between ROP17 expression and LC3B, Bcelin 1, p62, Bcl-2 and phosto-Bcl-2 were analysed using Pearson's correlation coefficient. $r$ $\geq 0.5$ was considered as strong correlation, and an $r<0.5$ was as poor correlation, $p<0.05$ was considered as statistically significant.

(Yamamoto et al. 2009, Ong et al. 2010). ROP18 phosphorylates immunity-related GTPases (IRGs) to avoid their accumulation in the PV and to protect the parasite from clearance (Fentress et al. 2010). ROP17 has a C-terminal kinase catalytic domain, and this kinase region contains a divergent $\mathrm{RAH}$ region that interacts with the parasitophorous vacuole membrane (PVM) of T. gondii (Reese and Boothroyd 2009). In infected cells, ROP17 resides on the cytosol side of the PVM and protects the parasite from clearance by forming complexes with ROP18 kinases that inhibit IRG recruitment to the intracellular T. gondii-containing vacuole (Etheridge et al. 2014). ROP17 inhibits the innate immune response by reprogramming host gene expression to promote $T$. gondii survival (Li et al. 2019). In addition, ROP17 has also been found to participate in the translocation of dense granule effectors across the PVM and promote $T$. gondii dissemination by hijacking monocyte tissue migration (Drewry et al. 2019, Panas et al. 2019).

Although some functions of ROP17 have been revealed, there are only a few reports on its function in parasite survival. In the present work, autophagy level was measured in $T$. gondii-infected murine models. Specifically, a ROP17-containing eukaryotic expression plasmid was transfected into HEK $293 \mathrm{~T}$ cells, and the regulatory role of ROP17 on autophagy was studied. Beclin 1 and LC3B are markers of autophagy since beclin 1 is involved in the initial step of autophagosome formation and LC3 forms puncta on the autophagosome membrane (Lopez Corcino et al. 2019). In addition, the ubiquitin-binding protein $\mathrm{p} 62$, which functions as a molecular adaptor for autophagic machinery and its substrates, has been widely used as a biochemical marker for general autophagy detection (Kraft et al. 2010). In the mouse model infected via intraperitoneal injection of tachyzoites of $T$. gondii, the levels of autophagy markers (LC3B, Beclin 1 and P62) were measured in small intestine tissues since $T$. gondii infects individual humans through the ingestion of contaminated feline faecal material, food and water, and first invades digestive tracts.

The immunohistochemistry data showed that LC3B and Beclin 1 were increased while p62 was decreased with the prolonged infection time. These data demonstrated that autophagy was initiated after infection with $T$. gondii. Given that autophagy has dual roles in $T$. gondii infection, either clearing this protozoan or providing nutrients for its proliferation (Portillo et al. 2010, 2017). Increasing evidence indicates that autophagy is essential for the elimination of T. gondii via canonical (Portillo et al. 2017, Lopez Corcino et al. 2019) or noncanonical autophagy (Selleck et al. 2015). To reveal the role of autophagy on the tachyzoites of $T$. gondii in the mouse intestine, immunohistochemistry was performed using rabbit anti-ROP17 serum, and the data showed that the number of parasites increased accompanied with the increase in autophagy markers expression.

To determine the role and mechanism of ROP17 in autophagy, the p3×Flag-VMV-14/ROP17 eukaryotic expression plasmid was transfected into HEK 293T cells, and autophagy was measured under serum starvation conditions since nutrient status is closely associated with autophagy activation. Morphologically, autophagy is char- 
acterised by the formation of double-membrane vesicles called autophagosomes, which are essential for the isolation and degradation of cytoplasmic components (Ryabovol and Minibayeva 2016). In addition, the number of GFP-LC3B puncta is a good indicator of autophagosome presence and is closely related to the number of autophagosomes (Meng et al. 2018). Our results showed that ROP17 markedly increased the number of autophagosomes in the ROP17-transfected cells, as demonstrated by the visualisation of MDC staining, number of GFP-LC3B puncta and TEM assessment. Western blotting also showed that the autophagy markers LC3B and Beclin 1 were increased in the ROP17-transfected cells compared to their levels in the control cells. Importantly, convincing evidence of autophagy activation with decreased p62 levels was detected by immunoblotting, which leads to the exclusion of failed autophagosome clearance as an explanation.

Beclin 1 plays an important role in autophagosome formation by interacting with Vps34 (also named class IIItype phosphoinositide 3-kinase) (Kihara et al. 2001). The ability of Beclin 1 to activate Vps34 is tightly regulated via both transcriptional regulation and interaction with Beclin 1-binding proteins (Maejima et al. 2016). Emerging evidence demonstrates that the binding of Bcl-2 to Beclin 1 negatively regulates autophagosome formation and then inhibits autophagy (Pattingre et al. 2005). Beclin 1 activates autophagy upon its dissociation from Bcl-2, which is phosphorylated by JNK (Young et al. 2013). Because ROP17 contains conserved ATP-binding residues and catalytic triads that are essential for $\mathrm{S} / \mathrm{T}$ kinases, similar to JNK (Qiu et al. 2009), we assayed the role of ROP17 in the phosphorylation of Bcl-2. Our results showed that ROP17 can interact with Bcl-2 and then phosphorylate it at Ser70 in the ROP17-transfected cells under starvation conditions. However, the levels of JNK expression and phosphorylation were unchanged under the same conditions.

Currently, the underlying molecular mechanisms for the effect of ROP17 on the virulence of $T$. gondii remain to be further investigated. Our findings suggest that ROP17 is advantageous to the survival and proliferation of the intracellular protist T. gondii via Beclin 1-Bcl-2-dependent autophagy activation. Our findings are consistent with previous reports that ROP17 functions as a virulent factor. Other studies show that ROP17 promotes the survival of $T$. gondii via inhibits the innate immune response of host cells ( $\mathrm{Li}$ et al. 2019) or ROP17 promotes dissemination of T. gondii by hijacking monocyte tissue migration (Drewry et al. 2019, Olafsson and Barragan 2020). Bcl-2 is also involved in the regulation of apoptosis, which is essential for the fate of $T$. gondii. Therefore, additional studies, including deletion of rop 17 gene in $T$. gondii or introduction of GFP/ RFP - ROP17 into T. gondii, are necessary to investigate the role of ROP17 in the crosstalk between components of autophagy and apoptosis to uncover its virulence function.

Acknowledgements. We would like to thank the American Journal Experts (AJE) for editing our manuscript. This work was supported by the Fund Program for the Scientific Activities of Selected Returned Overseas Professionals in Shanxi Province (No. 2014779), Shanxi Key Laboratory of Laboratory Animal Science and Experimental Animal Model of Human Diseases (No. 201901) and the Natural Science Foundation of Shanxi Province (No. 201801D121208)

\section{REFERENCES}

Chen L., Jiang H., Zhou Z., Li K., Deng G.Y., Liu Z. 2004 Purification of vitellin from the ovary of Chinese mitten-handed crab (Eriocheir sinensis) and development of an antivitellin ELISA. Comp. Biochem. Physiol. B Biochem. Mol. Biol. 138: 305-311.

Drewry L.L., Jones N.G., Wang Q., Onken M.D., Miller M.J., Sibley L.D. 2019: The secreted kinase ROP17 promotes Toxoplasma gondii dissemination by hijacking monocyte tissue migration. Nat. Microbiol. 4: 1951-1963.

DUBEY J.P. 1997a: Bradyzoite-induced murine toxoplasmosis: stage conversion, pathogenesis, and tissue cyst formation in mice fed bradyzoites of different strains of Toxoplasma gondii. J. Eukaryot. Microbiol. 44: 592-602.

DuBEY J.P. 1997b: Distribution of tissue cysts in organs of rats fed Toxoplasma gondii oocysts. J. Parasitol. 83: 755-757.

El Haju H., Demey E., Poncet J., Lebrun M., Wu B., Galeotti N., Fourmaux M.N., Mercereau-Puijalon O., Vial H., Labesse G., Dubremetz J.F. 2006: The ROP2 family of Toxoplasma gondii rhoptry proteins: proteomic and genomic characterization and molecular modeling. Proteomics 6: 5773-5784.

El Haju H., Lebrun M., Arold S.T., Vial H., Labesse G., DuBREMETZ J.F. 2007: ROP18 is a rhoptry kinase controlling the intracellular proliferation of Toxoplasma gondii. PLoS Pathog. 3: e14.

Etheridge R.D., Alaganan A., Tang K., Lou H.J., Turk B.E., Sibley L.D. 2014: The Toxoplasma pseudokinase ROP5 forms complexes with ROP18 and ROP17 kinases that synergize to control acute virulence in mice. Cell Host Microbe 15: 537-550.
Evans R.J., Sundaramurthy V., Frickel E.M. 2018: The interplay of host autophagy and eukaryotic pathogens. Front. Cell. Dev. Biol. 6: 118.

Fentress S.J., Behnke M.S., Dunay I.R., Mashayekhi M., Rommereim L.M., Fox B.A., Bzik D.J., Taylor G.A., Turk B.E., Lichti C.F., Townsend R.R., Qiu W., Hui R., Beatty W.L., Sibley L.D. 2010: Phosphorylation of immunity-related GTPases by a Toxoplasma gondii-secreted kinase promotes macrophage survival and virulence. Cell Host Microbe 8: 484-495.

Gao D., Zhang J., ZhaO J., Wen H., Pan J., Zhang S., Fang Y., Li X., CAi Y., Wang X., Wang S. 2014: Autophagy activated by Toxoplasma gondii infection in turn facilitates Toxoplasma gondii proliferation. Parasitol. Res. 113: 2053-2058.

Gomes L.C., Dikic I. 2014: Autophagy in antimicrobial immunity. Mol. Cell 54: 224-233.

Haкimi M.A., Olias P., Sibley L.D. 2017: Toxoplasma effectors targeting host signaling and transcription. Clin. Microbiol. Rev. 30: $615-645$.

Hassan E.A., Hassan M.H., Torad F.A. 2018: Correlation between clinical severity and type and degree of pectus excavatum in twelve brachycephalic dogs. J. Vet. Med. Sci. 80: 766-771.

Hermanns T., Muller U.B., Konen-Waisman S., Howard J.C., Steinfeldt T. 2016: The Toxoplasma gondii rhoptry protein ROP18 is an Irga6-specific kinase and regulated by the dense granule protein GRA7. Cell. Microbiol. 18: 244-259.

KAYE A. 2011: Toxoplasmosis: diagnosis, treatment, and prevention in congenitally exposed infants. J. Pediatr. Health Care. 25: 355-364. 
Kihara A., Noda T., Ishihara N., Ohsumi Y. 2001: Two distinct Vps34 phosphatidylinositol 3-kinase complexes function in autophagy and carboxypeptidase Y sorting in Saccharomyces cerevisiae. J. Cell. Biol. 152: 519-530.

Kraft C., Peter M., Hofmann K. 2010: Selective autophagy: ubiquitin-mediated recognition and beyond. Nat. Cell. Biol. 12: $836-841$.

Li J.X., He J.J., Elsheikha H.M., Chen D., Zhai B.T., Zhu X.Q., YAn H.K. 2019: Toxoplasma gondii ROP17 inhibits the innate immune response of HEK293T cells to promote its survival. Parasitol. Res. 118: 783-792.

Lopez Corcino Y., Gonzalez Ferrer S., Mantilla L.E., Trikeriotis S., Yu J.S., Kim S., Hansen S., Portillo J.C., Subauste C.S. 2019: Toxoplasma gondii induces prolonged host epidermal growth factor receptor signalling to prevent parasite elimination by autophagy: perspectives for in vivo control of the parasite. Cell. Microbiol. 21: e13084.

Ma G.Y., Zhang J.Z., Yin G.R., Zhang J.H., Meng X.L., ZhaO F. 2009: Toxoplasma gondii: proteomic analysis of antigenicity of soluble tachyzoite antigen. Exp. Parasitol. 122: 41-46.

Maejima Y., Isobe M., Sadoshima J. 2016: Regulation of autophagy by Beclin 1 in the heart. J. Mol. Cell. Cardiol. 95: 19-25.

Meng D., Li Z., Wang G., Ling L., Wu Y., Zhang C. 2018 Carvedilol attenuates liver fibrosis by suppressing autophagy and promoting apoptosis in hepatic stellate cells. Biomed. Pharmacother. 108: 1617-1627.

Montoya J.G., Liesenfeld O. 2004: Toxoplasmosis. Lancet 363: 1965-1976.

Olafsson E.B., Barragan A. 2020: The unicellular eukaryotic parasite Toxoplasma gondii hijacks the migration machinery of mononuclear phagocytes to promote its dissemination. Biol. Cell. 112: 239-250.

Ong Y.C., Reese M.L., Boothroyd J.C. 2010: Toxoplasma rhoptry protein 16 (ROP16) subverts host function by direct tyrosine phosphorylation of STAT6. J. Biol. Chem. 285: 28731-28740.

Panas M.W., Ferrel A., Naor A., Tenborg E., Lorenzi H.A., Boothroyd J.C. 2019: Translocation of dense granule effectors across the parasitophorous vacuole membrane in Toxoplas$m a$-infected cells requires the activity of ROP17, a rhoptry protein kinase. mSphere 4: e00276-19.

Pattingre S., Tassa A., Qu X., Garuti R., Liang X.H., Mizushima N., Packer M., Schneider M.D., Levine B. 2005: Bcl-2 antiapoptotic proteins inhibit Beclin 1-dependent autophagy. Cell 122: 927-939.

Peixoto L., Chen F., Harb O.S., Davis P.H., Beiting D.P., Brownback C.S., Ouloguem D., Roos D.S. 2010: Integrative genomic approaches highlight a family of parasite-specific kinases that regulate host responses. Cell Host Microbe 8: 208-218.

Portillo J.A., Okenka G., Reed E., Subauste A., Van Grol J., Gentil K., Komatsu M., Tanaka K., Landreth G., Levine B., Subauste C.S. 2010: The CD40-autophagy pathway is needed for host protection despite IFN-Gamma-dependent immunity and CD40 induces autophagy via control of P21 levels. PLoS One. 5: e14472.

Portillo J.C., Muniz-Feliciano L., Lopez Corcino Y., Lee S.J., Van Grol J., Parsons S.J., Schiemman W.P., Subauste C.S. 2017: Toxoplasma gondii induces FAK-Src-STAT3 signaling during infection of host cells that prevents parasite targeting by autophagy. PLoS Pathog. 13: e1006671.

Qiu W., Wernimont A., Tang K., Taylor S., Lunin V., Schapira M., Fentress S., Hui R., Sibley L.D. 2009: Novel struc- tural and regulatory features of rhoptry secretory kinases in Toxoplasma gondii. EMBO J. 28: 969-979.

Reese M.L., Boothroyd J.C. 2009: A helical membrane-binding domain targets the Toxoplasma ROP2 family to the parasitophorous vacuole. Traffic 10: 1458-1470.

Ryabovol V.V., Minibayeva F.V. 2016: Molecular mechanisms of autophagy in plants: role of ATG8 proteins in formation and functioning of autophagosomes. Biochemistry (Moscow) 81: 348-363.

SaeiJ J.P., Frickel E.M. 2017: Exposing Toxoplasma gondii hiding inside the vacuole: a role for GBPs, autophagy and host cell death. Curr. Opin. Microbiol. 40: 72-80.

Sanecka A., Frickel E.M. 2012: Use and abuse of dendritic cells by Toxoplasma gondii. Virulence 3: 678-689.

Santiago-Tirado F.H., Doering T.L. 2017: False friends: phagocytes as Trojan horses in microbial brain infections. PLoS Pathog. 13: e1006680.

Santos J.M., Soldati-Favre D. 2011: Invasion factors are coupled to key signalling events leading to the establishment of infection in apicomplexan parasites. Cell. Microbiol. 13: 787-796.

Schneider C.A., Figueroa Velez D.X., Azevedo R., Hoover E.M., Tran C.J., Lo C., Vadpey O., Gandhi S.P., Lodoen M.B. 2019: Imaging the dynamic recruitment of monocytes to the blood-brain barrier and specific brain regions during Toxoplasma gondii infection. Proc. Natl. Acad. Sci. USA 116: $24796-$ 24807.

Selleck E.M., Orchard R.C., Lassen K.G., Beatty W.L., Xavier R.J., Levine B., Virgin H.W., Sibley L.D. 2015: A noncanonical autophagy pathway restricts Toxoplasma gondii growth in a strain-specific manner in IFN-gamma-activated human cells. mBio 6: e1157-01115.

Subauste C.S. 2019: Interplay between Toxoplasma gondii, autophagy, and autophagy proteins. Front. Cell. Infect. Microbiol. 9: 139.

Taylor S., Barragan A., Su C., Fux B., Fentress S.J., Tang K., Beatty W.L., Haju H.E., Jerome M., Behnke M.S., White M., Wootton J.C., Sibley L.D. 2006: A secreted serine-threonine kinase determines virulence in the eukaryotic pathogen Toxoplasma gondii. Science 314: 1776-1780.

Van Grol J., Muniz-Feliciano L., Portillo J.A., Bonilha V.L., Subauste C.S. 2013: CD40 induces anti-Toxoplasma gondii activity in nonhematopoietic cells dependent on autophagy proteins. Infect. Immun. 81: 2002-2011.

Wang H.L., Wang Y.J., Pei Y.J., Bai J.Z., Yin L.T., Guo R., YIN G.R. 2016: DNA vaccination with a gene encoding Toxoplasma gondii rhoptry protein 17 induces partial protective immunity against lethal challenge in mice. Parasite 23: 4 .

Wang H.L., Zhang T.E., Yin L.T., Pang M., Guan L., Liu H.L., Zhang J.H., Meng X.L., Bai J.Z., Zheng G.P., Yin G.R. 2014: Partial protective effect of intranasal immunization with recombinant Toxoplasma gondii rhoptry protein 17 against toxoplasmosis in mice. PLoS One 9: e108377.

Wirawan E., Vanden Berghe T., Lippens S., Agostinis P., Vandenabeele P. 2012: Autophagy: for better or for worse. Cell Res. 22: 43-61.

Yamamoto M., Standley D.M., Takashima S., Saiga H., Okuyama M., Kayama H., Kubo E., Ito H., Takaura M., Matsuda T., Soldati-Favre D., Takeda K. 2009: A single polymorphic amino acid on Toxoplasma gondii kinase ROP16 determines the direct and strain-specific activation of Stat3. J. Exp. Med. 206: 2747-2760

Young M.M., Kester M., Wang H.G. 2013: Sphingolipids: regulators of crosstalk between apoptosis and autophagy. J. Lipid. Res. 54: 5-19. 